ISSN: $2317-8957$

Volume 6, Number 1, Jun. 2018

\title{
APLICAÇÃO DA TRIETILAMINA COMO INIBIDOR DE CORROSÃO DO AÇO API EM MEIO DE CLORETO
}

\author{
KRISHNA A. DA SILVA ${ }^{1}$, MAURÍCIO DOS S. VASCONCELLOS ${ }^{1}$, FRANCIELLY DE M. SANTOS ${ }^{1}$, \\ NEYDA DE LA C. OM TAPANES ${ }^{1}$, WILMA C. DE L. PINTO ${ }^{1}$, ANA ISABEL DE C. SANTANA ${ }^{1}$
}

${ }^{1}$ Fundação Centro Universitário Estadual da Zona Oeste

\section{RESUMO}

A corrosão é um processo de degradação dos materiais metálicos que ocorre em função da interação do metal ou liga metálica com o ambiente. Os inibidores de corrosão são conhecidos por ser amplamente utilizados como método para a redução ou inibição da corrosão. Os inibidores orgânicos são chamados de inibidores de adsorção, pois se adsorvem sobre a superfície metálica, promovendo a formação de uma barreira protetora entre o metal e o meio corrosivo. Em geral moléculas orgânicas contendo átomos de enxofre, nitrogênio e oxigênio são aplicadas como inibidores de adsorção. Neste trabalho foi avaliado o potencial inibidor da trietilamina sobre a corrosão do aço API em meio de $\mathrm{Nacl}(0,1 \mathrm{~mol} / \mathrm{L})$ e $\mathrm{HCl}(0,1 \mathrm{~mol} / \mathrm{L})$. Foi verificado que o efeito inibidor da trietilamina é mais significativo em meio de cloreto de sódio.

Palavras-chave: inibidor de corrosão, trietilamina, aço API, curva de polarização, ensaios gravimétricos.

\begin{abstract}
Corrosion is a process of degradation of metallic materials that occurs as a function of the interaction of the metal or metallic alloy with the environment. Corrosion inhibitors are widely employed as a method for reducing or inhibiting corrosion. Organic compounds are called adsorption inhibitors because they adsorb on the metal surface by forming a protective barrier between metals and corrosive enviroment. In general organic molecules containing sulfur, nitrogen and oxygen atoms are applied as adsorption inhibitors. In this work the triethylamine inhibitor potential on the corrosion of the API steel in $\mathrm{Nacl}(0.1 \mathrm{~mol} / \mathrm{L})$ and $\mathrm{HCl}(0.1 \mathrm{~mol} / \mathrm{L})$ medium was evaluated. It has been found that the inhibitory effect of triethylamine is more significant in sodium chloride medium.
\end{abstract}

Keywords: corrosion inhibitors, triethylamine, API steel, polarization curves, gravimetrics tests.

\section{INTRODUÇÃO}

A corrosão é um processo de degradação que ocorre espontaneamente (por ação química ou eletroquímica) e é atualmente correspondente a uma parcela considerável de deterioração dos materiais metálicos. A degradação de materiais causada pelos processos corrosivos provoca danos em estruturas e equipamentos acarretando prejuízos de ordem econômica e ambiental (Gentil 1996; Nunes 2007). A degradação de equipamentos e estruturas metálicas em virtude da corrosão ocorre em diferentes segmentos industriais, tais como: indústria de petróleo e gás, 
petroquímicas, alimentícia, naval, nuclear, na construção civil, na indústria automobilística, entre outras (Gentil 1996; Abboud et al. 2009).

Devido aos altos custos financeiros gerados pela corrosão e a crescente preocupação com os danos ambientais causados pelos processos corrosivos, cada vez mais se faz necessário investimentos em pesquisa e desenvolvimento acerca de métodos para a redução/ inibição dos processos corrosivos. Os inibidores de corrosão encontram-se entre os métodos mais utilizados para a inibição e/ou redução da degradação dos materiais metálicos em virtude da corrosão (Abboud et al. 2009; RameshKumar et al. 2015; Muthukumar et al. 2009; Obot et al. 2015; Albuquerque et al. 2015). As substâncias classificadas como inibidoras de corrosão são aquelas que ao serem adicionadas ao meio corrosivo promovem a redução/inibição da corrosão naquele meio oxidante específico (Albuquerque et al. 2015). O inibidor atua na redução da velocidade de corrosão do metal ou liga metálica (Singh et al. 2015), e é classificado em geral de acordo com o modo de atuação em anódico, catódico ou de adsorção. Os inibidores de adsorção vêm sendo amplamente avaliados na literatura, nestes casos a redução da corrosão ocorre em virtude da adsorção das moléculas dos inibidores, sobre a superfície ativa do metal (Singh et al. 2015; Al-Amiery et al. 2016; Khadiri et al. 2016; Yurt et al. 2014; Raghavendra \& Bhat, 2016; El-Lateef, 2015; Zhou et al. 2018). O fenômeno de adsorção sobre a superfície metálica pode ocorrer por quimiossorção ou por fisiossorção. A adsorção das moléculas promove um bloqueio dos sítios ativos, acarretando a formação um filme compacto sobre superfície metálica capaz de reduzir a taxa de corrosão (Obot et al. 2015; El-Rehim et al. 2010; Hmamoud et al. 2015). Uma vez que a eficiência de um inibidor de adsorção está relacionada com a estrutura da molécula inibidora, a carga da superfície metálica e com o tipo de eletrólito presente no meio corrosivo (Obot et al. 2015; El-Rehim et al. 2010), os compostos orgânicos que apresentam em sua estrutura grupos funcionais com a presença de heteroatomos tais como:
$\mathrm{N}, \mathrm{O}$ e $\mathrm{S}$, e de elétrons $\pi$ (ligações múltiplas) são conhecidos por apresentarem uma maior eficiência de inibição (Albuquerque et al. 2015; Singh et al. 2015; Hmamoud et al. 2015; Torres et al. 2016; Ngobiri et al. 2015).

Pesquisas apontam que moléculas com grupos funcionais tais como $-\mathrm{NH}_{2},-\mathrm{N}=\mathrm{N}, \mathrm{C}=\mathrm{N}$, -CNS, CHO, N-H são conhecidos por apresentarem propriedades inibidoras da corrosão em diferentes meios (El-Lateef 2015; Khaled \& Hackerman 2003). Dentre as classes de compostos orgânicos recentemente avaliadas como inibidoras de corrosão podem citar as bases de Schiff. Esses compostos se adsorvem formando uma monocamada sobre a superfície metálica (El-Lateef, 2015).

As aminas e seus derivados também são amplamente utilizados como inibidores de corrosão em virtude de serem relativamente fáceis de sintetizar, possuírem alta solubilidade em meio ácido e boa eficiência de inibição (Muthukumar et al. 2009; Zhou et al. 2018; Khaled \& Hackerman 2003; Gupta et al. 2012; El-haddad \& Fouda 2015; Galal et al. 2005; Kuang et al. 2014; Hegazy \& Aiad 2015; Martínez et al. 2009; Zhang et al. 2015; Ansari et al. 2015; Yadav et al. 2016). A capacidade inibidora da corrosão dos sais de amônio e compostos aromáticos também foram avaliadas (Fouda et al. 2017; Guo et al. 2015). E mais recentemente muitas pesquisas estão sendo direcionadas para a avaliação da atividade inibidora de corrosão de compostos à base de extratos vegetais (Shabani-Nooshabadi \& Ghandchi 2015; Assis et al. 2015; De Barros et. al. 2015; Anjos et al. 2013). A utilização na indústria dos extratos vegetais como inibidores de corrosão ainda não está sendo plenamente realizada, no entanto grandes esforços estão sendo realizados a fim de obter inibidores ambientalmente amigáveis a partir dos extratos vegetais.

Considerando os custos gerados pelos processos corrosivos, a ampla aplicação de compostos com grupamento amino como inibidores de corrosão e a relação custo benefício desses inibidores, este trabalho teve como objetivo avaliar a ação da trietilamina (amina de 
cadeia aberta) como inibidor da corrosão do aço API 5L em em meio contendo íons cloreto em diferentes $\mathrm{pH}(\mathrm{NaCl} 0,1 \mathrm{~mol} \mathrm{~L}-1)$ e $(\mathrm{HCl} 0,1$ mol L-1).

\section{MATERIAIS E MÉTODOS}

A amostra metálica utilizada para a confecção dos corpos de prova foi o aço API 5L com composição química, em peso ( $\%)(0,122 \%$ C, $0,920 \% \mathrm{Mn}, 0,182 \% \mathrm{Si}, 0,026 \% \mathrm{Al}, 0,005 \%$ S, $0,009 \mathrm{Ni}, 0,008 \% \mathrm{Mo}, 0,021 \% \mathrm{Cu}, 0,002 \%$ $\mathrm{P}, 0,003 \% \mathrm{Ni}+\mathrm{Ti}+\mathrm{V}$, balanço $\mathrm{Fe}$ ). O efeito da adição da trietilamina sobre a corrosão do aço nos meios de $\mathrm{HCl}$ 0,1 mol L-1 e $\mathrm{NaCl}$ 0,1 mol $\mathrm{L}^{-1}$ foi avaliado através de ensaios eletroquímicos e gravimétricos.

Para a realização dos ensaios gravimétricos, as amostras foram usinadas na forma de coupons com área de $8,35 \mathrm{~cm}^{2}$. Após a usinagem foi feito no topo das amostras um furo com 1,0 mm de diâmetro. Para os ensaios eletroquími$\cos$ foi feito na amostra de aço um contato elétrico com fio de cobre e em seguida foi obtido um embutimento em resina epóxi. A usinagem dos corpos de prova foi obtida com auxílio de cortadora metalográfica (TECLAGO - modelo CM60). Após a usinagem as amostras foram lixadas com lixas de diferentes granulometrias (100, 200, 300, 400 e 600 mesh), em politriz de bancada (Arotec - modelo AROPOL 2V). Em seguida, os corpos de prova foram lavados com água destilada, desengordurados com álcool e secos com jato de ar frio.

As soluções utilizadas como meio eletrolíticos foram preparadas com água destilada e reagente de grau analítico (VETEC). Todos os ensaios foram realizados em meio aerado e em temperatura ambiente. Quatro diferentes concentrações de trietilamina foram adicionadas às soluções utilizadas como meio eletrolítico conforme apresentado na Tabela 1.
Tabela 1: Condições com e sem a presença do inibidor.

\begin{tabular}{cc}
\hline Condição & Concentração $\left(\mathrm{mol} \mathrm{L}^{-1}\right)$ \\
\hline 1 & $\mathrm{NaCl} 0,1 \mathrm{M}$ \\
2 & $\mathrm{NaCl}+1 \times 10^{-2}$ Trietilamina \\
3 & $\mathrm{NaCl}+1 \times 10^{-3}$ Trietilamina \\
4 & $\mathrm{NaCl}+5 \times 10^{-3}$ Trietilamina \\
5 & $\mathrm{NaCl}+7 \times 10^{-3}$ Trietilamina \\
6 & $\mathrm{HCl} 0,1$ M \\
7 & $\mathrm{HCl}+1 \times 10^{-2}$ Trietilamina \\
8 & $\mathrm{HCl}+1 \times 10^{-3}$ Trietilamina \\
9 & $\mathrm{HCl}+5 \times 10^{-3}$ Trietilamina \\
10 & $\mathrm{HCl}+7 \times 10^{-3}$ Trietilamina \\
\hline
\end{tabular}

A avaliação do comportamento cinético do aço utilizado foi realizada através de ensaios eletroquímicos. Os ensaios eletroquímicos foram obtidos em um potenciostato/galvanostato (AUTOLAB, modelo PGSTAT 302N), em cada uma das condições apresentadas na Tabela 1. Foi utilizada uma célula convencional de três eletrodos: contra-eletrodo (eletrodo de platina), eletrodo de referência (calomelano) e eletrodo de trabalho (aço API). Os ensaios consistiram na realização de medida de potencial aberto por um período de $3600 \mathrm{~s}$ e posterior obtenção das curvas de polarização no modo potenciodinâmico, utilizando uma velocidade de varredura de 1 $\mathrm{mV} / \mathrm{s}$. Os experimentos foram realizados até a obtenção de uma triplicata para cada condição ensaiada.

O ensaio de imersão (ensaio gravimétrico) foi obtido mediante a imersão das amostras metálicas nos meios corrosivos com e sem a adição de trietilamina. Antes da imersão no meio eletrolítico a massa inicial dos corpos de prova foi obtida em balança analítica de quatro dígitos. Em solução de $\mathrm{NaCl}$ as amostras foram imersas por um período de $2 \mathrm{~h}, 4 \mathrm{~h}, 6 \mathrm{~h}, 24 \mathrm{~h}, 48 \mathrm{~h}$, $72 \mathrm{~h}, 96 \mathrm{~h}$ e $168 \mathrm{~h}$. No ensaio em meio de $\mathrm{HCl}$, a imersão foi realizada no período de $2 \mathrm{~h}, 4 \mathrm{~h}$, $6 \mathrm{~h}, 24 \mathrm{~h}, 48 \mathrm{~h}$ e $72 \mathrm{~h}$. Os ensaios foram realiza- 
dos em triplicata. Após cada período de imersão foi realizada a limpeza das amostras seguida de pesagem para a obtenção da perda de massa. Os ensaios de imersão foram realizados tendo como base as normas ASTM G1 e G31.

A partir dos dados obtidos através do ensaio de imersão, foi possível calcular as taxas de corrosão e eficiência de inibição de acordo com as equações 1 e 2 , respectivamente:

$$
v=\frac{k W}{A t \rho}
$$

Onde, (1):

$\mathrm{v}$ : Taxa de corrosão

$\mathrm{k}$ : constante $(87600 \mathrm{~mm} / \mathrm{ano})$

W: Perda de massa em g

A: área da amostra em $\mathrm{cm}^{2}$

T: Tempo de exposição em horas

$\rho$ : Massa específica do material em $\mathrm{g} / \mathrm{cm}^{3}$

$$
\bar{n} \%=\frac{W_{0}-W}{W_{0}}
$$

Onde (2):

n\%: Eficiência de corrosão

W0: Taxa de inibição sem inibidor

W: Taxa de inibição com inibidor

\section{RESULTADOS E DISCUSSÃO Ensaios Eletroquímicos}

Os ensaios eletroquímicos realizados neste trabalho consistiram na avaliação da evolução do potencial de circuito aberto do aço API e de curvas de polarização potenciodinâmicas obtidas em ambos os meios corrosivos $(\mathrm{NaCl}$ e $\mathrm{HCl})$ com e sem adição de trietilamina. Os ensaios eletroquímicos permitem avaliar a cinética das reações anódicas e catódicas que ocorrem sobre a superfície da amostra metálica e auxiliam na compreensão do comportamento do inibidor (Obot et al. 2015, Hegazy \& Aiad 2015, Shabani-Nooshabadi \& Ghandchi 2015). As Figuras 1 e 2 apresentam a evolução de potencial de circuito aberto do aço API 5L nos mei- os eletrolíticos avaliados. É possível observar que a adição de trietilamina à solução contendo $\mathrm{NaCl}$ (Figura 1), provoca um deslocamento do potencial de circuito aberto do aço para valores mais nobres. Observa-se que essa tendência se acentua com o aumento da concentração da trietilamina. Este comportamento sugere que no meio corrosivo avaliado $\left(\mathrm{NaCl} 0,1 \mathrm{~mol} \mathrm{~L}^{-1}\right.$, meio aerado e temperatura ambiente) a adição da trietilamina possui um maior controle sobre o processo anódico, ou seja, sobre a oxidação do metal, logo, atua sobre a reação de dissolução metálica (corrosão). Um comportamento semelhante foi verificado por Shabani-Nooshabadi e Ghandchi (2015), Obot et al. (2015) e Hegazy e Aiad (2015) ao avaliar diferentes tipos de inibidores, que relacionaram o deslocamento do potencial de circuito aberto para valores mais nobres à adsorção das moléculas do inibidor sobre a superfície, o que acarretaria numa diminuição dos processos corrosivos.

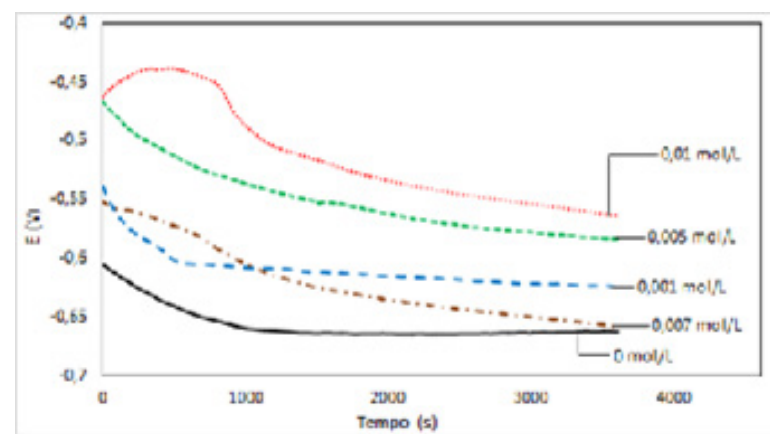

Figura 1: Evolução de potencial de circuito aberto para o aço API na ausência e presença de diferentes concentrações da trietilamina, a $25^{\circ} \mathrm{C} \mathrm{em} \mathrm{NaCl} 0,1 \mathrm{~mol} / \mathrm{L}$.

A evolução de potencial de circuito aberto do aço API em meio de $\mathrm{HCl} 0,1 \mathrm{~mol} \mathrm{~L}^{-1}$ com e sem adição do inibidor pode ser observado na Figura 2. Diferentemente do que se verifica para o meio contendo $\mathrm{NaCl}$, em condições de acidez elevada a adição de trietilamina, em $\mathrm{HCl}$ não provocou alterações significativas no potencial de circuito aberto do aço. 


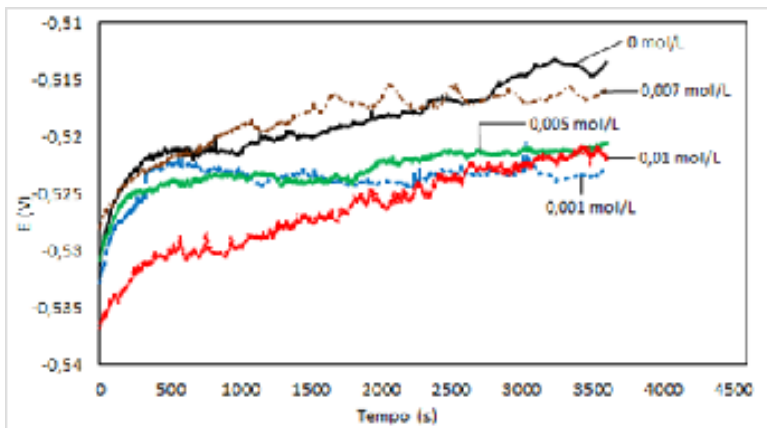

Figura 2: Evolução de potencial de circuito aberto para o aço API na ausência e presença de diferentes concentrações da trietilamina, a $25^{\circ} \mathrm{C}$ em $\mathrm{HCl} 0,1 \mathrm{~mol} / \mathrm{L}$.

Contrariamente do que se observa em meio básico, a adição do inibidor em meio ácido não promoveu um deslocamento significativo do potencial de corrosão. Nas situações nas quais o deslocamento ocorreu, verifica-se que o potencial foi deslocado para valores mais negativos. Este comportamento sugere que em meio fortemente ácido, a trietilamina afeta de forma mais significativa a reação catódica que ocorre sobre a superfície metálica.

As curvas de polarização potenciodinâmicas obtidas para o aço API em meio de $\mathrm{NaCl}$ podem ser verificadas na Figura 3.

Observa-se que em meio de cloreto, a adição da trietilamina provoca um deslocamento significativo do potencial de corrosão $\left(\mathrm{E}_{\text {corr }}\right)$ do aço, em todas as concentrações de inibidor testadas. Esse comportamento corrobora os resultados observados para o potencial de circuito aberto $\left(\mathrm{E}_{\mathrm{pca}}\right)$ nas mesmas condições. Além disso, verifica-se uma redução considerável da corrente no ramo anódico das curvas, onde se observa inclusive uma região na qual a corrente deixa de variar linearmente com o potencial (Figura 3). Esse comportamento sugere um bloqueio da superfície metálica, ou seja, a adição de trietilamina nas condições avaliadas, provocou a formação de um filme sobre a superfície do aço, promovendo uma redução dos processos anódicos (dissolução metálica), o que acarreta em uma diminuição do processo corrosivo.

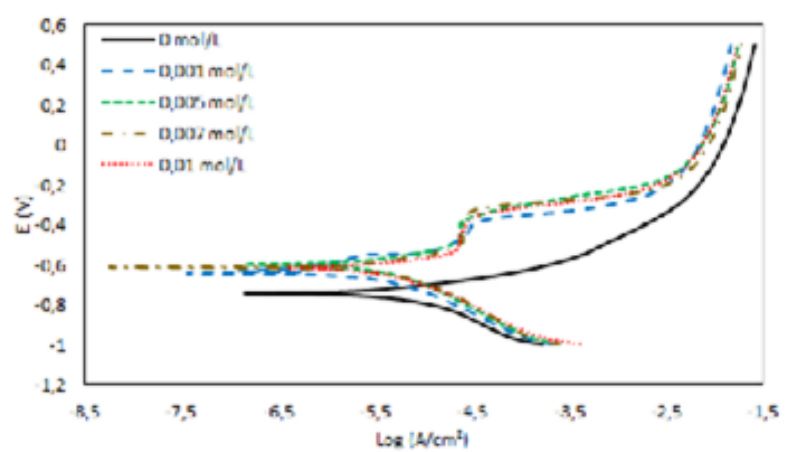

Figura 3: Curvas de Polarização para o aço API na ausência e presença de diferentes concentrações da trietilamina, a $25^{\circ} \mathrm{C}$ em NaCl 0,1 mol/L.

Do mesmo modo que observado para o potencial de circuito aberto, as curvas de polarização obtidas para o aço em meio ácido $(0,1$ $\mathrm{M}$ de $\mathrm{HCl}$ ) apresentadas na Figura 4, mostram que a adição do inibidor não exerceu influência significativa sobre as regiões anódica e catódica das curvas. Verifica-se que para potenciais mais elevados ( $\mathrm{E}>-300 \mathrm{mV}$ ) observa-se que a adição do inibidor provocou uma leve redução da corrente no ramo anódico. Este comportamento pode estar associado com a maior agressividade do meio eletrolítico, que poderia afetar a cinética das reações químicas. Ou seja, nestas condições a adição de trietilamina não promove uma redução efetiva dos processos corrosivos que ocorrem sobre a superfície do material.

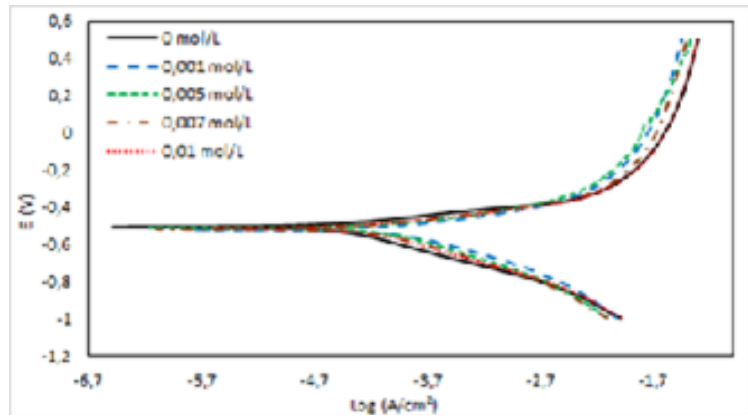

Figura 4: Curvas de Polarização para o aço API na ausência e presença de diferentes concentrações da trietilamina, a $250 \mathrm{C} \mathrm{em} \mathrm{HCl} \mathrm{0,1} \mathrm{mol/L.}$

\section{Ensaios gravimétricos (de imersão)}

A partir dos ensaios de imersão foi possível obter os valores de perda de massa (obtidos em triplicata), e através destes calcular a taxa de corrosão, a eficiência de inibição e a fra- 
ção da superfície metálica recoberta pelas moléculas do inibidor.

Os dados de perda de massa apresentaram uma boa reprodutibilidade e desta forma, a média das triplicatas foi utilizada para o cálculo das taxas de corrosão e da eficiência de inibição, apresentados nas Figuras 5 e 6 para a imersão em do aço em meio de $\mathrm{NaCl}$.

Verifica-se claramente que a adição de trietilamina provoca uma redução da taxa de corrosão do aço API, sobretudo nas primeiras horas de imersão. Após períodos mais longos, a taxa de corrosão do material atinge valores muito próximos tanto na presença quanto na ausência do inibidor. Não foi possível verificar uma relação linear entre a concentração do inibidor e a taxa de corrosão do aço. Contudo, embora não se verifique uma relação linear, observa-se claramente que a redução na taxa de corrosão e o aumento na eficiência de inibição tornam-se mais significativos com o aumento da concentração da trietilamina.

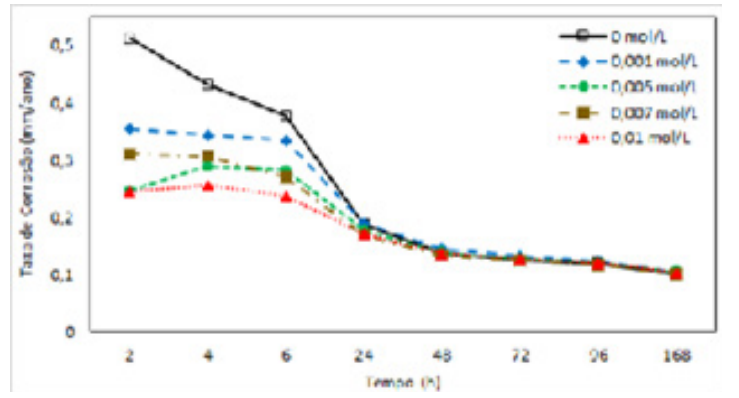

Figura 5: Taxa de corrosão do aço API na ausência e presença de diferentes concentrações da trietilamina, a $25^{\circ} \mathrm{C}$ em $\mathrm{NaCl} \mathrm{0,1} \mathrm{mol} / \mathrm{L}$.

Da mesma forma, não foi possível observar uma relação clara entre a concentração e a eficiência do inibidor. No entanto se verifica que a maior eficiência de inibição foi observada para a maior concentração avaliada ( $1 \times 10^{-2} \mathrm{~mol} / \mathrm{L}$ de trietilamina). Foi possível verificar também que a maior eficiência ocorre nas primeiras horas de imersão, para todas as concentrações de inibidor avaliadas.

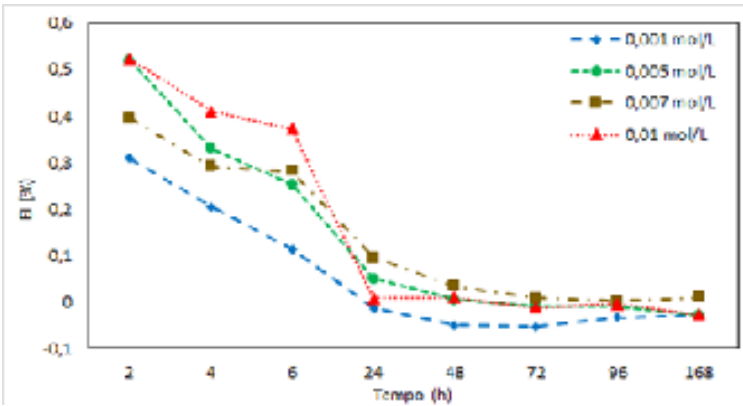

Figura 6: Eficiência de inibição no aço API na presença de diferentes concentrações da trietilamina, a $25^{\circ} \mathrm{C}$ em $\mathrm{NaCl}$ 0,1 mol/L.

A Figura 7 apresenta as taxas de corrosão calculadas para o aço API em meio de 0,1 $\mathrm{M} \mathrm{HCl}$ com e sem a presença do inibidor.

Observa-se claramente que a adição da trietilamina provoca uma diminuição da taxa de corrosão em todas as concentrações avaliadas, e esta redução se mantêm praticamente constante durante todo o tempo de imersão. No entanto, mesmo em presença do inibidor, as taxas de corrosão em meio de $\mathrm{HCl}$ são cerca de 10 vezes mais elevadas que em meio de $\mathrm{NaCl}$. Este comportamento pode estar relacionado com a maior agressividade do meio eletrolítico, que mesmo em presença do inibidor ainda permanece com taxas de corrosão mais elevadas. Do mesmo modo quem em meio de $\mathrm{NaCl}$, não foi possível observar uma relação clara entre a taxa de corrosão, a eficiência de inibição e a concentração do inibidor. No entanto, se verifica claramente que a menor taxa de corrosão ocorreu quando a amostra metálica esteve imersa na menor concentração de trietilamina $\left(1 \times 10^{-3} \mathrm{~mol} / \mathrm{L}\right)$.

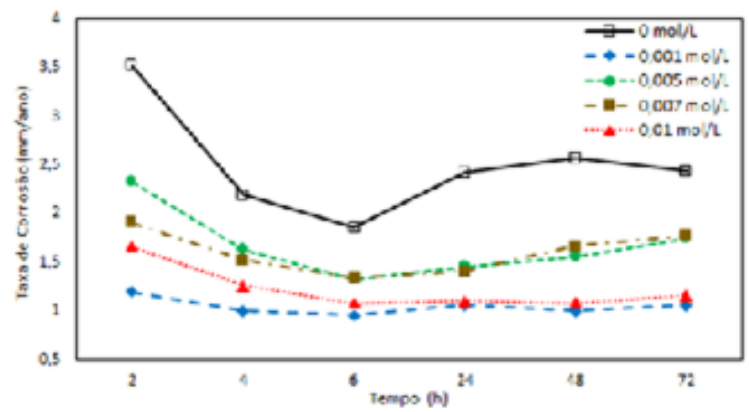

Figura 7: Taxa de corrosão do aço API na ausência e presença de diferentes concentrações da trietilamina, a $25^{\circ} \mathrm{C}$ em $\mathrm{HCl} 0,1 \mathrm{~mol} / \mathrm{L}$. 
Os resultados da eficiência de inibição ( $)$ e da fração de recobrimento da superfície $(\theta)$, podem ser observados na Figura 8. Como já era esperado a maior eficiência e consequentemente a maior fração de cobertura é verificada para a menor concentração de trietilamina. Do mesmo modo que em meio de $\mathrm{NaCl}$, a maior eficiência de inibição foi observada nas primeiras horas de imersão.

Os resultados obtidos a partir dos ensaios gravimétricos mostram que apesar de não ter sido observado a partir das curvas de polarização um efeito inibidor significativo da trietilamina em meio de $\mathrm{HCl}$, esse composto pode atuar na inibição da corrosão do aço API em solução fortemente ácida. A diferença observada entre os ensaios gravimétricos e eletroquímicos para o meio de $\mathrm{HCl}$, pode estar relacionado com a polarização da superfície nos ensaios eletroquímicos.

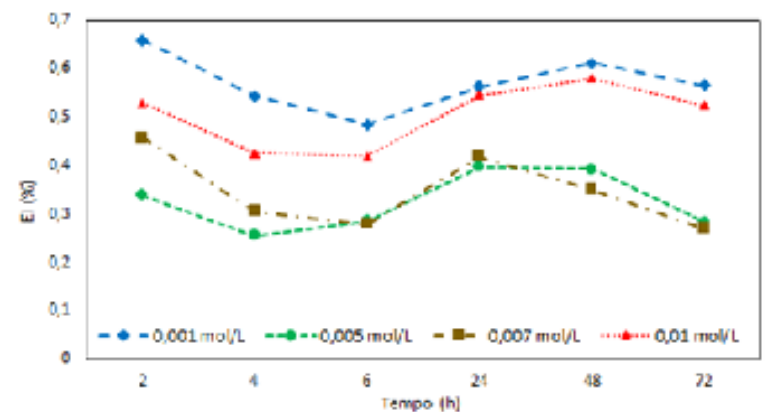

Figura 8: Eficiência de inibição no aço API na presença de diferentes concentrações da trietilamina, a $25^{\circ} \mathrm{C}$ em

$$
\mathrm{NaCl} 0,1 \mathrm{~mol} / \mathrm{L} \text {. }
$$

\section{Isotermas de Adsorção}

As Figuras 9 e 10 apresentam as isotermas de adsorção de Langmuir ( $\mathrm{C} / \theta$ versus $\mathrm{C})$, obtidos dos dados experimentais da perda de massa. Através do modelo matemático de Langmuir, foram obtidos os valores de correlação $\left(\mathrm{R}^{2}\right)$ para cada ajuste. É possível observar que o melhor ajuste foi obtido para os ensaios realizados em meio de $\mathrm{NaCl}$. Através do coeficiente linear da reta, foi possível calcular o valor da constante de adsorção $\left(\mathrm{K}_{\text {ads }}\right)$

A constante de adsorção está relacionada com o parâmetro termodinâmico da energia livre através da equação 3:

$$
\Delta G_{a d s}^{o}=-R T \ln \left(55.5 K_{a d s}\right)
$$

Onde, $\mathrm{R}$ é a constante dos gases $(8,314$ $\mathrm{J} / \mathrm{mol}$ ), T é a temperatura termodinâmica do sistema (Kelvin) e 55,5 corresponde à concentração da água em solução (mol/L).

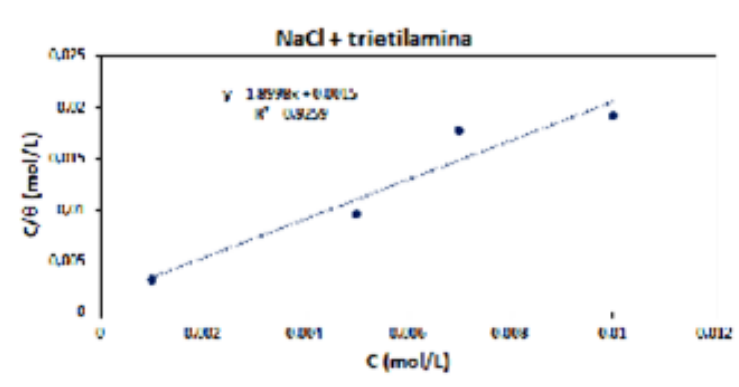

Figura 9: Isoterma de Adsorção de Langmuir para a adsorção da trietilamina sobre o aço API, a $25^{\circ} \mathrm{C}$ em meio de $\mathrm{NaCl}(0,1 \mathrm{~mol} / \mathrm{L})$

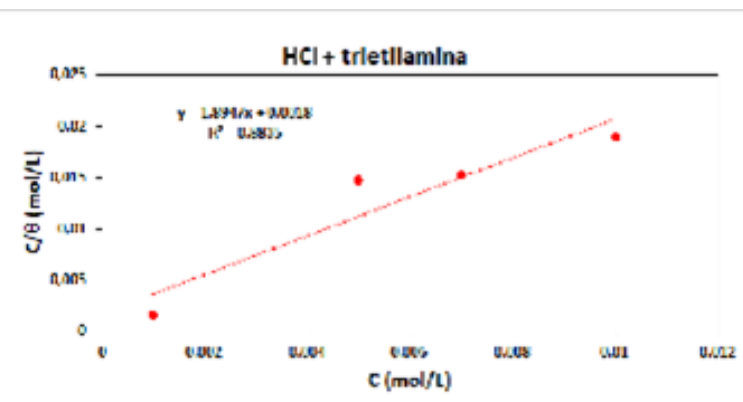

Figura 10: Isoterma de Adsorção de Langmuir para a adsorção da trietilamina sobre o aço API, a $25^{\circ} \mathrm{C}$ em meio de $\mathrm{HCl}(0,1 \mathrm{~mol} / \mathrm{L})$

Partindo dos dados obtidos através da equação da reta na Figuras 9 e 10, foi possível calcular os valores da constante de adsorção e da energia livre relacionada ao processo, conforme apresentados na Tabela 2.

Tabela 2: Valores termodinâmicos para o processo de adsorção da trietilamina sobre o aço API

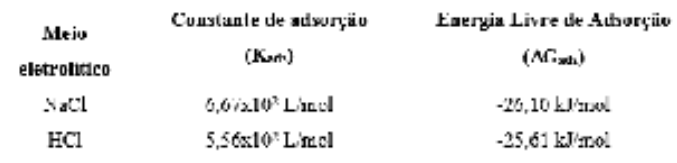

Verifica-se mediante os dados obtidos que a interação entre a trietilamina e a superfície do aço API é semelhante para ambos os 
meios avaliados $(\mathrm{HCl}$ e $\mathrm{NaCl})$, neste caso é possível afirmar que o $\mathrm{pH}$ do meio não exerce influência significativa sobre o processo de adsorção. Os valores negativos de $\Delta \mathrm{G}_{\text {ads }}$ indicam que o processo de adsorção da trietilamina sobre o aço API é espontâneo em ambos os meios avaliados.

Em geral considera-se que valores (em módulo) de $\Delta \mathrm{G}_{\mathrm{ads}}$ próximos ou maiores que 40 $\mathrm{kJ} / \mathrm{mol}$ expressam um processo de quimiossorção (El-Rehim et al. 2010; Hmamoud et al. 2015), onde a interação entre as moléculas e a superfície do metal são fortes, da ordem de ligação covalente. Por outro lado, valores próximos ou menores que $20 \mathrm{~kJ} / \mathrm{mol}$ representam um processo de fisissorção, onde a interação é do tipo eletrostática (Obot et al. 2015; Zhang et al. 2015; Shabani-Nooshabadi \& Ghandchi 2015; Assis et al. 2015; Singh et al. 2015). Considerando os valores obtidos para a trietilamina em $\mathrm{NaCl}\left(\Delta \mathrm{G}_{\mathrm{ads}}=-26,10 \mathrm{~kJ} / \mathrm{mol}\right)$ e em $\mathrm{HCl}$ $\left(\Delta \mathrm{G}_{\mathrm{ads}}=-25,61 \mathrm{~kJ} / \mathrm{mol}\right)$ é possível afirmar que o processo é de fisissorção, ou seja, a adsorção ocorre através de interações eletrostáticas entre as moléculas carregadas do inibidor e a superfície metálica. Essa afirmação pode explicar porque a maior inibição ocorre nas primeiras horas de imersão. Uma vez que a fisissorção é um processo de adsorção menos intenso que a quimiossorção, a intensidade de recobrimento da superfície metálica diminui com o tempo.

\section{Avaliação da superfície após ensaios de imersão}

A evolução dos processos corrosivos sobre a superfície metálica com e sem a presença do inibidor foi avaliada durante a evolução dos ensaios gravimétricos, conforme pode ser observado nas macrografias apresentadas nas Figuras 11 e 12, obtidas nos diferentes meios com e sem a presença da trietilamina $\left(0,01 \mathrm{~mol} . \mathrm{L}^{-1}\right)$.
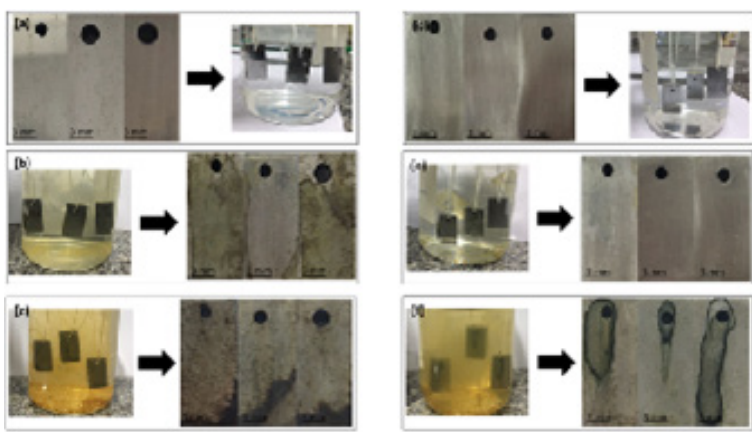

Figura 11: Macrografias das amostras do aço imersas em $\mathrm{NaCl}$ 0,1 M (a - 0h, b - 2h, c - 24h) e NaCl com presença da Trietilamina $1 \times 10^{-2} \mathrm{~mol} / \mathrm{L}(\mathrm{d}-0 \mathrm{~h}, \mathrm{e}-2 \mathrm{~h}, \mathrm{f}-24 \mathrm{~h})$
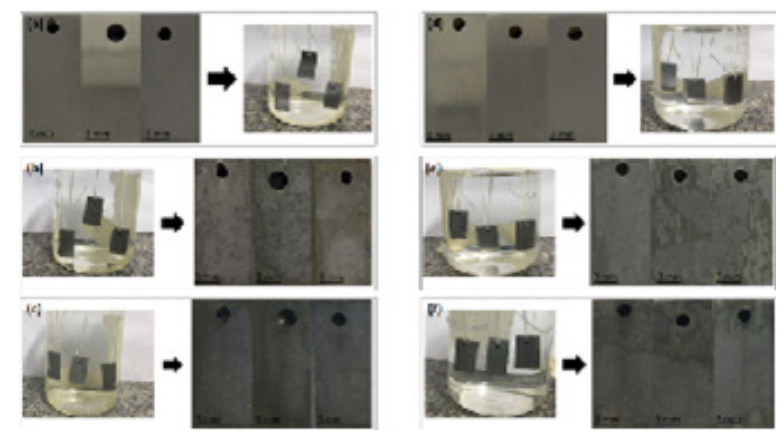

Figura 12: Macrografias imersas em $\mathrm{HCl} 0,1 \mathrm{M}(\mathrm{a}-0 \mathrm{~h}, \mathrm{~b}$ - 2h, c - 24h) e $\mathrm{HCl}$ com presença da Trietilamina $1 \times 10^{-2}$ $\mathrm{mol} / \mathrm{L}(\mathrm{d}-0 \mathrm{~h}, \mathrm{e}-2 \mathrm{~h}, \mathrm{f}-24 \mathrm{~h})$

Considerando as macrografias apresentadas acima se verifica que a adição da trietilamina promove uma diminuição dos processos corrosivos observados sobre a superfície metálica. A inibição é mais facilmente perceptível em meio de $\mathrm{NaCl}$, onde se verifica na ausência do inibidor uma maior quantidade de produtos de corrosão (Figuras $11 \boldsymbol{b}$ e $\boldsymbol{c}$ ). Após a adição de trietilamina, é possível verificar uma diminuição desses produtos de corrosão (Figura $11 \boldsymbol{e}$ e f). A inibição é mais facilmente observada para as primeiras horas de imersão. Esse resultado corrobora com o que foi observado em termos de eficiência de inibição, onde se verificou que o máximo de eficiência ocorre nas primeiras horas de imersão.

Para as amostras imersas em meio de $\mathrm{HCl}$, não se verificam grandes diferenças nos processos corrosivos sobre a superfície das amostras metálicas. Entretanto, se observa para as primeiras horas de imersão uma pequena diferença entre as amostras imersas no meio com e sem adição de trietilamina (Figuras $12 \boldsymbol{b}$ 
e $\boldsymbol{e})$. Esse comportamento era esperado em virtude da maior agressividade da solução de $\mathrm{HCl}$.

\section{CONCLUSÃO}

Mediante os resultados obtidos, foi possível concluir que nas condições experimentais avaliadas:

- Os resultados eletroquímicos mostraram que a adição de trietilamina em meio de $\mathrm{NaCl}$ promove um efeito mais significativo sobre os processos anódicos que ocorrem na superfície do aço API. Em meio de $\mathrm{HCl}$ não foram verificados resultados significativos nos ensaios eletroquímicos. Esse comportamento confirma que o tipo de eletrólito exerce influência na ação da trietilamina como inibidor de corrosão.

- Os ensaios gravimétricos mostraram que a adição de trietilamina provocou a redução da taxa de corrosão em ambos os meios eletrolíticos em todas as concentrações aplicadas. A redução foi mais significativa nas primeiras horas de imersão, tanto em meio de $\mathrm{NaCl}$, quanto em meio de $\mathrm{HCl}$. Entretanto, as taxas de corrosão em presença de $\mathrm{HCl}$ são mais elevadas em comparação com as amostras imersas em $\mathrm{NaCl}$. - $\quad$ Não foi possível verificar uma relação linear entre a eficiência de inibição e a concentração do inibidor para ambos os meios avaliados. Entretanto, em solução de $\mathrm{NaCl}$ observase uma tendência de aumento da eficiência com aumento da concentração de trietilamina.

- Verificou-se que o processo de adsorção da trietilamina sobre o aço API se dá por fisiossorção, que consiste em uma interação eletrostática entre as moléculas da trietilamina e a superfície do aço. A adsorção por fisiossorção foi observada tanto em $\mathrm{NaCl}$ quanto em $\mathrm{HCl}$.

\section{REFERÊNCIAS}

ABBOUD Y, ABOURRICHE A, SAFFAJ T, BERRADA M, CHARROUF M, BENNAMARA A, HANNACHE H. 2009. A novel azo dye, 8-quinolinol-5-azoantipyrine as corrosion inhibitor for mild steel in acidic media. Desalination 237: 175-189.

ANJOS GC, ALMEIDA CC, MELO DMA, MARTINEZ-HUITLE CA, ROSSI CGFT, MACIEL MAM. 2013. Eficiência de Anacardium occidentale Linn em um Sistema Microemulsionado na Inibição a Corrosão de Aço Carbono. Rev. Virtual Quim. 5 (4): 760-769.

AL-AMIERY A, KASSIM F, KADHUM A, MOHAMAD A. 2016. Synthesis and characterization of a novel eco-friendly corrosion inhibition for mild steel in $1 \mathrm{M}$ hydrochloric acid, Sci. Rep. 6.

ALBUQUERQUE M, OLIVEIRA M, ECHEVARRIAA. 2015. Avaliação da Atividade Anticorrosiva de Formulações com Extrato Vegetal por Técnicas Eletroquímicas e Gravimétrica. Rev. Virtual Quim. 7 (5): 1841-1853.

ANSARI KR, QURAISHI MA, SINGH A. 2015. Corrosion inhibition of mild steel in hydrochloric acid by some pyridine derivatives: An experimental and quantum chemical study. Journal of Industrial and Engineering Chemistry 25: 89-98.

ASSIS B, MEIRA F, PINA V, ANDRADE G, COTRIM B, RESENDE G, D'ELIA E, SOUZA F. 2015. Efeito Inibitório do Extrato de Piper Nigrum L. sobre a Corrosão do Aço Carbono em Meio Ácido. Revista Virtual Química: 1830-1840.

ASTM INTERNATIONAL G 1 - 03. 2017. Standard Practice for Preparing, Cleaning, and Evaluating Corrosion Test Specimens.

ASTM INTERNATIONAL G 31 - 72. 2004. Standard Practice for Laboratory Immersion 
Corrosion Testing of Metals.

DE BARROS IB, MOSCOSO HZL, CUSTODIO DL, VEIGA JUNIOR VF, BASTOS IN. 2015. Casca Preciosa (Aniba canelilla) como Inibidor de Corrosão do Aço-Carbono. Rev. Virtual Quim. 7 (5): 1743-1755.

EL-HADDAD MN, FOUDA AS. 2015. Electroanalytical, quantum and surface characterization studies on imidazole derivatives as corrosion inhibitors for aluminum in acidic media. Journal of Molecular Liquids 209: 480-486.

EL-LATEEF H. 2015. Experimental and computational investigation on the corrosion inhibition characteristics of mild steel by some novel synthesized imines in hydrochloric acid solutions. Corros. Sci. 92: 104-117.

EL-REHIM S, SAYYAH S, EL-DEEB M, KAMAL S, AZOOZ R. 2010. Poly(o-phenylenediamine) as an inhibitor of mild steel corrosion in $\mathrm{HCl}$ solution. Materials Chemistry and Physics 123: 20-27.

FOUDA AS, ELMORSI MA, SHABAN SM, FAYED T, AZAZY O. 2017. Evaluation of N-(3-(dimethyl hexadecyl ammonio)propyl) palmitamide bromide as cationic surfactant corrosion inhibitor for API N80 steel inacidic environment. Egyptian Journal of Petroleum. In Press.

GALAL A, ATTA NF, AL-HASSAN MHS. 2005. Effect of some thiophene derivatives on the electrochemical behavior of AISI 316 austenitic stainless steel in acidic solutions containing chloride ions I. Molecular structure and inhibition efficiency relationship. Materials Chemistry and Physics 89: 38 - 48.

GUO L, ZHU S, ZHANG S. 2015. Experimental and theoretical studies of benzalkonium chloride as an inhibitor for carbon steel corrosion in sulfuric acid. Journal of Industrial and Engineering Chemistry 24: 174-180.
GUPTA M, BHARA K, SINGH G. 2012. N-ethyl N-hydroxyethyl aniline (NENHEA) as corrosion inhibitor for mild steel in $0.5 \mathrm{M}$ sulfuric acid. Corros. Prot. Mater. $31: \mathrm{n}^{\mathrm{o}} 1$.

HEGAZY MA, AIAD I. 2015. 1-Dodecyl4-(((3-morpholinopropyl)imino)methyl) pyridin-1-ium bromide as a novel corrosion inhibitor for carbon steel during phosphoric acid production, Journal of Industrial and Engineering Chemistry 31: 91-99.

HMAMOU D, SALGHI R, ZARROUK A, ZARROK H, TOUZANI R, HAMMOUTI A, ASSYRY EL. 2015. Investigation of corrosion inhibition of carbon steel in $0.5 \mathrm{M}$ $\mathrm{H} 2 \mathrm{SO} 4$ by new bipyrazole derivative using experimental and theoretical approaches. Journal of Environmental Chemical Engineering 3 (3): 2031-2041.

KHADIRI A, SADDIK R, BEKKOUCHE $\mathrm{K}$, AOUNITI A, HAMMOUT B, BENCHAT N, BOUACHRINE M, SOLMAZ R. 2016. Gravimetric, electrochemical and quantum chemical studies of so mepyridazine derivatives as corrosion inhibitors for mild steel in $1 \mathrm{M} \mathrm{HCl}$ solution. Journal of the Taiwan Institute of Chemical Engineers 58: 552-564.

KHALED KF, HACKERMAN N. 2003. Investigation of the inhibitive effect of orthosubstituted anilines on corrosion of iron in $1 \mathrm{M} \mathrm{HCl}$ solutions, Electrochim. Acta 48: 2715-2723.

KUANG W, MATHEWS JA, MACDONALD DD. 2014. The effect of Anodamine on the corrosion behavior of 1018 mild steelin deionized water: I. Immersion and polarization tests. Electrochimica Acta 127: $79-85$. 
MARTÍNEZ D, GONZALEZ R, MONTEMAYOR K, JUAREZ-HERNANDEZ A, FAJARDO G, HERNANDEZ-RODRIGUEZ MAL. 2009. Amine type inhibitor effect on corrosion-erosion wear in oil gas pipes. Wear 267: 255-258.

MUTHUKUMAR N, ILANGOVAN A, MARUTHAMUTHU S, PALANISWAMY N, KIMURA A. 2009. 1-Aminoanthraquinone derivatives as a novel corrosion inhibitor for carbon steel API 5L-X60 in white petrol-water mixtures. Materials Chemistry and Physics 115: 444-452.

NGOBIRI NC, OGUZIE EE, OFORKA NC, AKARANTA O. 2015. Comparative study on the inhibitive effect of Sulfadoxine-Pyrimethamine and an industrial inhibitor on the corrosion of pipeline steel in petroleum pipeline water. Arabian Journal of Chemistry. In Press.

OBOT IB, UMOREN S, GASEN Z, SULEIMAN R, BASSAM E. 2015. Theoretical prediction and electrochemical evaluation of vinylimidazole and allylimidazole as corrosion inhibitors for mild steel in $1 \mathrm{M} \mathrm{HCl}$. Journal of Industrial and Engineering Chemistry 21: 1328-1339.

RAGHAVENDRA N, BHAT J. 2016. Green approach to inhibition of corrosion of aluminum in $0.5 \mathrm{M} \mathrm{HCl}$ medium by tender arecanut seed extract: insight from gravimetric and electrochemical studies. Res. Chem. Intermed. 42 (7): 6351-6372.

RAMESHKUMAR S, DANAEE I, RASHVANDAVEI M, VIJAYAN M. 2015. Quantum chemical and experimental investigations on equipotent effects of $(+) \mathrm{R}$ and $(-) \mathrm{S}$ enantiomers of racemic amisulpride as eco-friendly corrosion inhibitors for mild steel in acidic solution. Journal of Molecular Liquids 212: 168-186.

SHABANI-NOOSHABADI M, GHANDCHI MS. 2015. Santolina chamaecyparissus extract as a natural source inhibitor for 304 stainless steel corrosion in $3.5 \% \mathrm{NaCl}$. Journal of Industrial and Engineering Chemistry 31: 231-237.

SINGH A, YUANHUA L, OBOT IB, EBENSO EE, ANSARI KR, QURAISHI MA. 2015. Corrosion mitigation of J55 steel in $3.5 \% \mathrm{NaCl}$ solution by a macrocyclic inhibitor. Applied Surface Science 356: 341-347.

SINGH MR, GUPTA P, GUPTA K. 2015. The litchi (Litchi Chinensis) peels extractas a potential green inhibitor in prevention of corrosion of mild steel in $0.5 \mathrm{M} \mathrm{H} 2 \mathrm{SO} 4$ solution. Arabian Journal of Chemistry. In Press.

TORRES V, CABRAL G, DA SILVA AC, FERREIRA KC, D'ELIA E. 2016. Ação inibidora de extratos da semente do mamão papaia na corrosão do aço-carbono $1020 \mathrm{em}$ hcl 1 mol 1-1. Quim. Nova 39 (4): 423-430.

YADAV M, BEHERA D, SHARMA U. 2016. Nontoxic corrosion inhibitors for N80 steel in hydrochloric acid. Arabian Journal of Chemistry 9: S1487-S1495.

YURT A, DURAN B, DAL H. 2014. An experimental and theoretical investigation on adsorption properties of some diphenolic Schiff bases as corrosion inhibitors at acidic solution/mild steel interface. Arabian Journal of Chemistry 7: 732-740.

ZHANG H, PANG X, ZHOU M, LIU C, WEI L, GAO K. 2015. The behavior of pre-corrosion effect on the performance ofimidazoline-based inhibitor in 3 wt. $\% \mathrm{NaCl}$ solution saturatedwith CO2. Applied Surface Science 356: 63-72.

ZHOU L, LV Y, HU Y, ZHAO J, XIA X, LI X. 2018. Experimental and theoretical investigations of 1,3,5-tris(4-aminophenoxy)benzene as an effective corrosion inhibitor for mild steel in $1 \mathrm{M} \mathrm{HCl}$. Journal of Molecular Liquids 249: 179-187. 